\title{
Indicadores de saúde da COVID-19 nos primeiros quatro meses no estado de São Paulo
}

RESUMO | Objetivo: Analisar a estimativa dos indicadores de saúde da COVID-19 nos quatro primeiros meses da pandemia a partir da confirmação do primeiro caso. Método: Estudo ecológico. Foram coletados os casos confirmados de COVID-19 do Estado de São Paulo (ESP) dos meses de fevereiro a junho, obtidos do Centro de Vigilância Epidemiológica do ESP. A análise dos dados foi realizada a partir de indicadores de saúde e a população foi obtida pela Fundação Sistema Estadual de Análise de Dados do ESP. O estudo não passou por Comitê de Ética e Pesquisa por se tratar de dados públicos. Resultado: Nos primeiros quatro meses da pandemia da COVID-19 no ESP houve aumento consecutivos do número de municípios afetados, casos confirmados, óbitos, coeficientes de incidência e mortalidade e declínio do coeficiente de letalidade. Conclusão: Verificamos diminuição dos óbitos da COVID-19 no ESP e isso pode estar associado ao aprimoramento do manejo clínico da doença.

Palavras-chaves: Infecções por Coronavírus; Pandemias; Epidemiologia Descritiva; Estudos Ecológicos.

\begin{abstract}
Objective: To analyze the estimate of the health indicators of COVID-19 in the first four months of the pandemic from the confirmation of the first case. Method: Ecological study. Confirmed cases of COVID-19 from the State of São Paulo (ESP) from February to June were collected from the Center for Epidemiological Surveillance of ESP. Data analysis was carried out based on health indicators and the population was obtained by the ESP State System of Data Analysis Foundation. The study did not go through the Ethics and Research Committee because it is public data. Result: In the first four months of the COVID-19 pandemic in ESP, there was a consecutive increase in the number of affected municipalities, confirmed cases, deaths, incidence and mortality rates and a decline in the lethality rate. Conclusion: We verified a decrease in the deaths of COVID-19 in the ESP and this may be associated with the improvement of the clinical management of the disease.
\end{abstract}

Keywords: Coronavirus Infections; Pandemics; Epidemiology Descriptive; Ecological Studies.

RESUMEN | Objetivo: Analizar la estimación de los indicadores de salud de COVID-19 en los primeros cuatro meses de la pandemia desde la confirmación del primer caso. Método: Estudio ecológico. Los casos confirmados de COVID-19 del Estado de São Paulo (ESP) de febrero a junio fueron recolectados del Centro de Vigilancia Epidemiológica de ESP. El análisis de los datos se realizó con base en indicadores de salud y la población fue obtenida por la Fundación Sistema Estatal de Análisis de Datos ESP. El estudio no pasó por el Comité de Ética e Investigación por tratarse de datos públicos. Resultado: En los primeros cuatro meses de la pandemia de COVID-19 en ESP, hubo un aumento consecutivo en el número de municipios afectados, casos confirmados, defunciones, tasas de incidencia y mortalidad y una disminución en la tasa de letalidad. Conclusión: Verificamos una disminución de las muertes por COVID-19 en el ESP y esto puede estar asociado a la mejora del manejo clínico de la enfermedad.

Palabras claves: Infecciones por Coronavirus; Pandemias; Epidemiología Descriptiva; Estudios Ecológicos.

\section{Roudom Ferreira Moura}

Enfermeiro, Doutorando do Programa de Epidemiologia da Faculdade de Saúde Pública Universidade de São Paulo, Técnico do Centro de Vigilância Epidemiológica "Prof. Alexandre Vranjac" e do Centro de Vigilância Sanitária Centro de Controle de Doenças - Secretaria de Estado da Saúde de São Paulo.

ORCID: 0000-0002-0685-4627

\section{Ana Paula Miranda Mundim-Pombo}

Enfermeira e Médica Veterinária, Doutora em Ciências pela Faculdade de Medicina da Universidade de São Paulo, Professora da Universidade São Judas Tadeu - São Paulo. São Paulo (SP). ORCID: 0000-0002-1877-0965

\section{Janessa de Fátima Morgado de Oliveira}

Farmacêutica-bioquímica, Doutora em Ciências pela Faculdade de Saúde Pública da Universidade de São Paulo, Investigadora - Departamento de Epidemiologia do Instituto de Saúde Pública da Universidade do Porto (Projeto DOCnet). Porto (Porto). ORCID: 0000-0002-8482-5872

\section{Rosângela Elaine Minéo Biagolini}

Enfermeira, Doutora em Ciências da Saúde pela Escola de Enfermagem da Universidade de São Paulo, Professora da Universidade Nove de Julho - São Paulo (SP). Enfermeira da Unidade de Vigilância em Saúde da Secretaria Municipal de Saúde de São Paulo (SP).

ORCID: 0000-0003-2061-5611

Recebido em: 28/08/2020

Aprovado em: 09/11/2020

INTRODUÇÃO

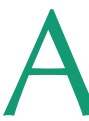
COVID-19 (do inglês: Coronavírus Disease 2019), causada pelo Novo Coronavírus (SARS-CoV-2), detectada em Wuhan na China, trata-se de uma doença emergente capaz de se disseminar intensamente e infectar milhões de pessoas ${ }^{1-3}$. Desde o primeiro caso em dezembro de 2019, a doença vem se disseminando de modo que a Organização Mundial da Saúde declarou em janeiro de 2020, o surto como emergência de saúde pública de interesse internacional ${ }^{4}$.

No Brasil, em fevereiro de 2020 o Ministério da Saúde declarou a COVID-19 como uma situação de emergência em saúde pública de importância nacional ${ }^{5}$, mas devido ao avanço e expansão em nível global, em março de 2020, foi declarada como pandemia 6 .

A disseminação do agente causal, SARS-CoV-2, e consequentemente da pandemia, avançou de modo que em 27 de setembro de 2020 foram confirmados 32.730 .945 casos e 991.224 mortes de COVID-19 no mundo. Essa casuística foi, principalmente, composta por notificações oriundas das regiões: Americana (16.233.110 casos/ 346.864 mortes); Europeia (5.562.875 ca- 
sos/ 234.681 mortes); Mediterrâneo Oriental (2.340.215 casos/ 60.345 mortes); Pacífico Ocidental (600.891 casos/ 13.129 mortes), Sudeste Asiático (6.720.771 casos/ 110.711 mortes) e Africana (1.172.342 casos/ 25.481 mortes) ${ }^{7}$.

A região das Américas, liderada pelos Estados Unidos e em segundo lugar o Brasil, foi a principal afetada no mundo, concentrando cerca de $50 \%$ dos casos e $55 \%$ das mortes. Só na semana de 21 a 27 de setembro essa região foi responsável por $52 \%$ das mortes mundiais ${ }^{7}$.

No Brasil, o monitoramento de casos e óbitos, realizado continuamente por meio da vigilância epidemiológica, registrou 4.717.991 casos e 141.406 mortes acumulados até 26 de setembro8. Mesmo com a orientação para adoção de medidas visando redução da infecção, a realidade do país nem sempre permite que as recomendações de distanciamento físico e higienização sejam respeitadas em localidades periféricas e nas moradias precárias, em que se mantem muitos residentes por cômodos e/ou em áreas onde o acesso à água limpa é difícil ou onde mesmo a necessidade do emprego informal impede de tomar as medidas preventivas cabíveis ${ }^{9-10}$.

Entre as principais regiões acometidas no Brasil, considerando os coeficientes de incidência por 100 mil habitantes até o dia 03 de outubro de 2020, destacaram-se o Centro-Oeste $(3.533,2)$, o Norte $(3.362,4)$ e o Nordeste $(2.282,7)$; já a região Sudeste apresentou o menor coeficiente de incidência $(1.862,2)$. Ao detalhar a situação da região Sudeste, o Espírito Santo registrou o maior coeficiente de incidência $(3.202,8)$ e na sequência São Paulo foi o segundo maior afetado $(2.114 .2)^{8}$.

Em relação à mortalidade, ao realizar a análise considerando também os coeficientes de mortalidade por $100 \mathrm{mil}$ habitantes, as regiões Norte, Centro-Oeste e Sudeste são predominantemente acometidas com 80,3; 75,3; e 72,4, respetivamente. No Estado de São Paulo, a capital paulistana ainda chama atenção pela frequência de casos e óbitos, apesar da tendência de estabilização esperada. A região sudeste e, principalmente, o Estado paulista vem sendo destaque desde a notificação do primeiro caso do Brasil e América Latina, em fevereiro de $2020^{8,11}$.

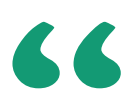

\section{A região das}

Américas, liderada pelos Estados Unidos e em segundo lugar o Brasil, foi a principal afetada no mundo, concentrando cerca de $50 \%$ dos casos e $55 \%$ das mortes. Só na semana de 21 a 27 de setembro essa região foi responsável por $52 \%$ das mortes mundiais.

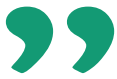

No Estado de São Paulo, pesquisas sobre a COVID-19 são necessárias porque se trata de uma doença emergente a partir de 2020. Além disso, ainda são poucos os estudos que realizaram análise dos indicadores de saúde dessa doença no país e no Estado de São Paulo. Também, mais especificamente, não foram encontradas tais análises para o Brasil e Estado de São Paulo para os primeiros quatro meses da pandemia. Investigar e compreender mais detalhadamente a epidemiologia da
COVID-19 no Estado de São Paulo gera a expectativa de poder contribuir, ao originar subsídios aos gestores de saúde para decisões em saúde pública.

Tendo em vista essas premissas, o presente estudo buscou respostas ao seguinte questionamento: como se deu a distribuição dos indicadores de saúde da COVID-19 no Estado de São Paulo nos quatro primeiros meses dessa pandemia?

Dessa forma, o objetivo deste estudo foi descrever os indicadores de saúde da COVID-19, de 26 de fevereiro a 26 de junho de 2020, que se constituíram nos quatro primeiros meses da pandemia no Estado de São Paulo.

\section{MÉTODOS}

Estudo epidemiológico, do tipo ecológico, de base populacional. A população do Estado de São Paulo foi objeto do estudo, considerando sua distribuição nos 645 municípios do Estado. A amostra foi constituída pelos casos confirmados de COVID-19, obtidos a partir da base de dados de domínio público do Governo do Estado de São Paulo - Secretaria de Estado da Saúde - Coordenadoria de Controle de Doenças - Centro de Vigilância Epidemiológica "Prof. Alexandre Vranjac" - Novo Coronavírus (COVID-19) - Situação Epidemiológica 26 de fevereiro a 26 de junho de 2020, disponíveis em: http://www. saude.sp.gov.br/cve-centro-de-vigilancia-epidemiologica-prof.-alexandre-vranjac/ areas-de-vigilancia/doencas-de-transmissao-respiratoria/coronavirus-covid-19/situacao-epidemiologica.

O primeiro mês, refere-se ao período de 26 de fevereiro a 25 de março. O segundo de 26 de março a 25 de abril. O terceiro de 26 de abril a 25 de maio e o quarto de 26 de maio a 26 de junho.

Para o cálculo dos indicadores de saúde: estimativas dos coeficientes de incidência e mortalidade foram utilizadas a população obtida do sítio eletrônico do Instituto Brasileiro de Geografia e Estatística (IBGE) 2019.

Para estimar os coeficientes de inci- 
dência e mortalidade por 100.000 habitantes foram calculados a partir da razão entre o número de casos confirmados e óbitos, respectivamente (numerador) e a população residente (denominador).

Para estimar o coeficiente de letalidade por 100 casos foi calculado a partir da razão entre o número de óbitos (numerador)

Figura 1 - Distribuição dos casos confirmados de COVID-19 (A - 1 mês após o primeiro caso confirmado; B - 2 meses após o primeiro caso; C - 3 meses após o primeiro caso e D - 4 meses após o primeiro caso), segundo município de residência. Estado de São Paulo, 26 de fevereiro a 26 de junho de 2020.

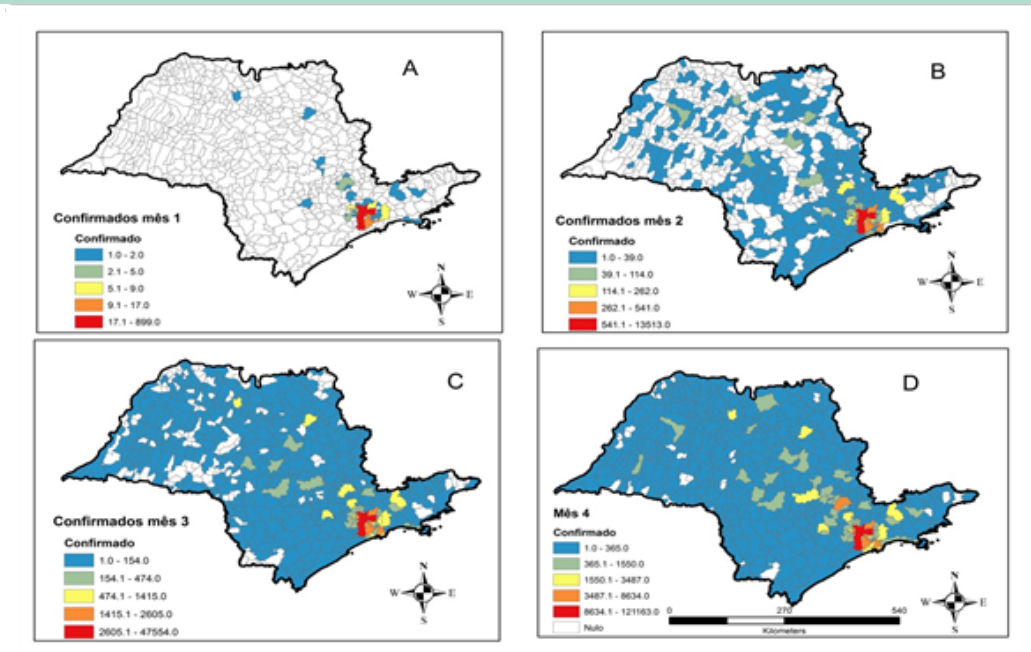

Fonte: Governo do Estado de São Paulo / Secretaria de Estado da Saúde / Coordenadoria de Controle de Doenças / Centro de Vigilância Epidemiológica "Prof. Alexandre Vranjac" - Novo Coronavírus (COVID-19) - Situação Epidemiológica.

Figura 2 - Distribuição do coeficiente de incidência (por 100.000 habitantes) de COVID-19 (A - 1 mês após o primeiro caso confirmado; B - 2 meses após o primeiro caso; $C-3$ meses após o primeiro caso e $D-4$ meses após o primeiro caso), segundo município de residência. Estado de São Paulo, 26 de fevereiro a 26 de junho de 2020.
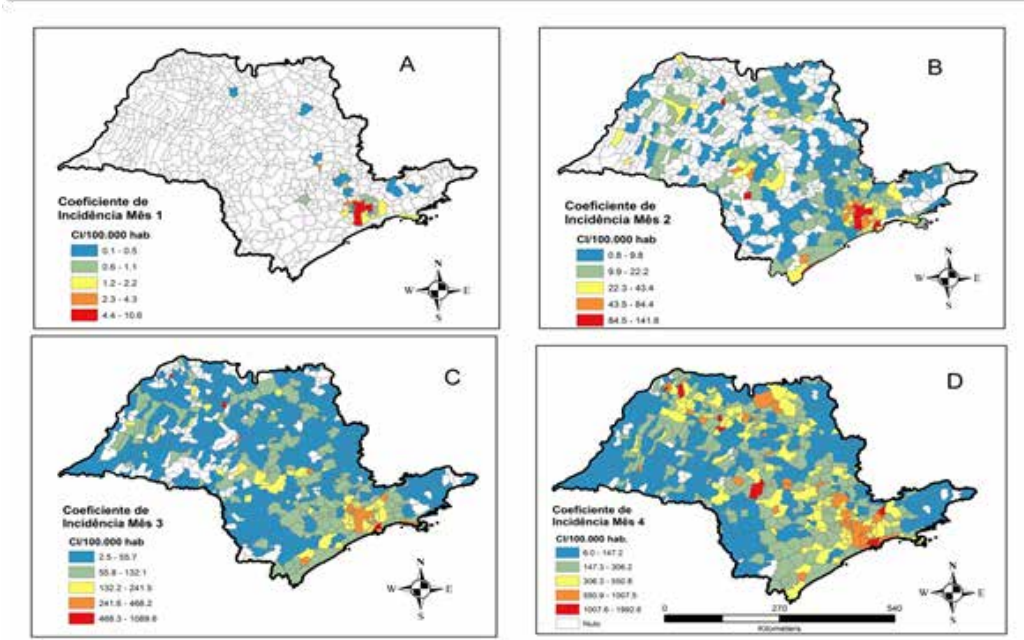

Fonte: Governo do Estado de São Paulo / Secretaria de Estado da Saúde / Coordenadoria de Controle de Doenças / Centro de Vigilância Epidemiológica "Prof. Alexandre Vranjac" - Novo Coronavírus (COVID-19) - Situação Epidemiológica / IBGE.

e os casos confirmados (denominador).

Para a análise dos dados, utilizou-se estatística descritiva, aplicando-se os Softwares TabWin 3.6b, Microsoft Excel 2016.

Para confecção dos mapas foi utilizado o software SIGs ArcGis versão 10.5 e Qgis10.

A pesquisa não precisou ser aprovada por Comitê de Ética de Pesquisa, haja vista que os dados secundários da COVID-19 obtidos para análise nesse estudo são de domínio público e não apresentou identificação dos pacientes, seguindo, assim, os princípios estabelecidos pela Resolução $n^{\circ}$ 466, de 12 de dezembro de 2012, que dispõe sobre as diretrizes e normas regulamentadoras de pesquisa envolvendo seres humanos.

\section{RESULTADOS}

A disseminação do SARS-CoV-2 teve início na capital do Estado de São Paulo, com a divulgação do primeiro caso pelo Centro de Vigilância Epidemiológica "Prof. Alexandre Vranjac" / Centro de Controle de Doenças / Secretaria de Estado da Saúde / Governo do Estado de São Paulo em 26 de fevereiro de 2020. O aumento do número de casos foi alarmante e após quatro meses da pandemia por COVID-19 (26 de junho de 2020), o Estado de São Paulo já havia acumulado 258.508 casos confirmados em 616 municípios (95,5\% dos 645 municípios), sendo o município de São Paulo o mais atingindo (46,9\%) (Figura 1).

Houve variação de $2.034,84 \%$ em relação à estimativa do coeficiente de incidência do primeiro mês $(2,21$ casos/100.000 habitantes) para o segundo (44,97 casos/100.000 habitantes), $8.421,27 \%$ do primeiro para o terceiro mês (186,11 casos/100.000 habitantes), e de $25.473,30 \%$ do primeiro para o quarto mês $(562,96$ casos por 100.000 habitantes) no Estado de São Paulo.

Os municípios com as maiores estimativas de risco, considerando-se todos os quatro meses de ocorrência da doença, foram Santos (1992,56 casos para cada 
100.000 habitantes), Igaratá (1520,87 casos para cada 100.000 habitantes), Cubatão $(1518,69$ casos para cada 100.000 habitantes), Macatuba $(1497,41$ casos para cada 100.000 habitantes) e Santa Salete $(1423,95$ casos para cada 100.000 habitantes) (Figura 2).

O primeiro óbito por COVID-19 foi
Figura 3 - Distribuição dos óbitos de COVID-19 (A - 1 mês após o primeiro caso confirmado; B - 2 meses após o primeiro caso; C -3 meses após o primeiro caso e D - 4 meses após o primeiro caso), segundo município de residência. Estado de São Paulo, 26 de fevereiro a 26 de junho de 2020.

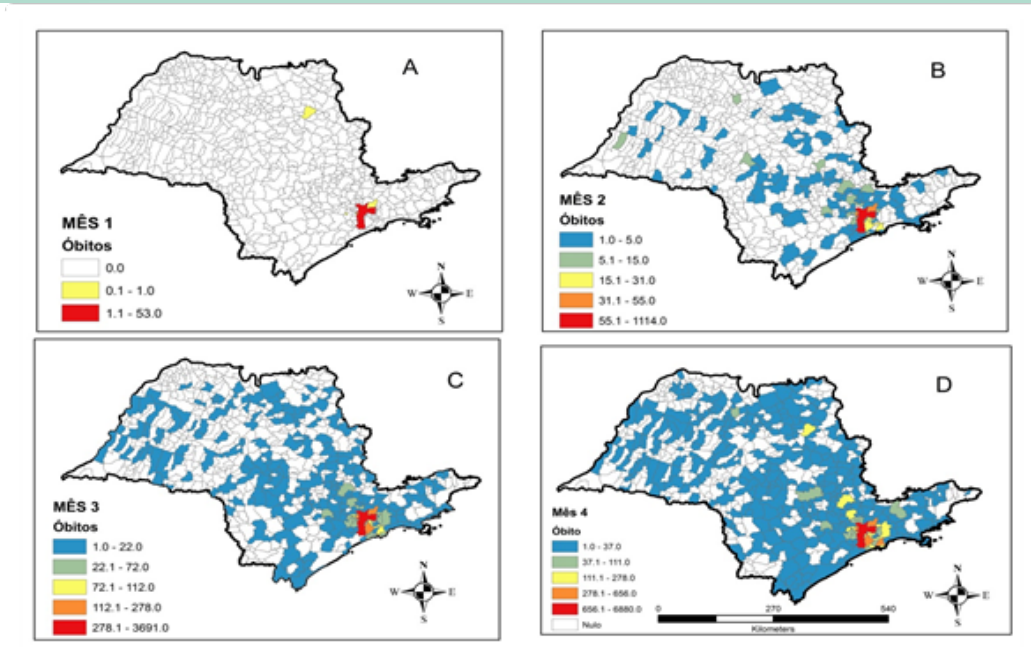

Fonte: Governo do Estado de São Paulo / Secretaria de Estado da Saúde / Coordenadoria de Controle de Doenças / Centro de Vigilância Epidemiológica "Prof. Alexandre Vranjac" - Novo Coronavírus (COVID-19) - Situação Epidemiológica.

Figura 4 - Distribuição do coeficiente de mortalidade (por 100.000 habitantes) de COVID-19 (A - 1 mês após o primeiro caso confirmado; B - 2 meses após o primeiro caso; C - 3 meses após o primeiro caso e D - 4 meses após o primeiro caso), segundo município de residência. Estado de São Paulo, 26 de fevereiro a 26 de junho de 2020.
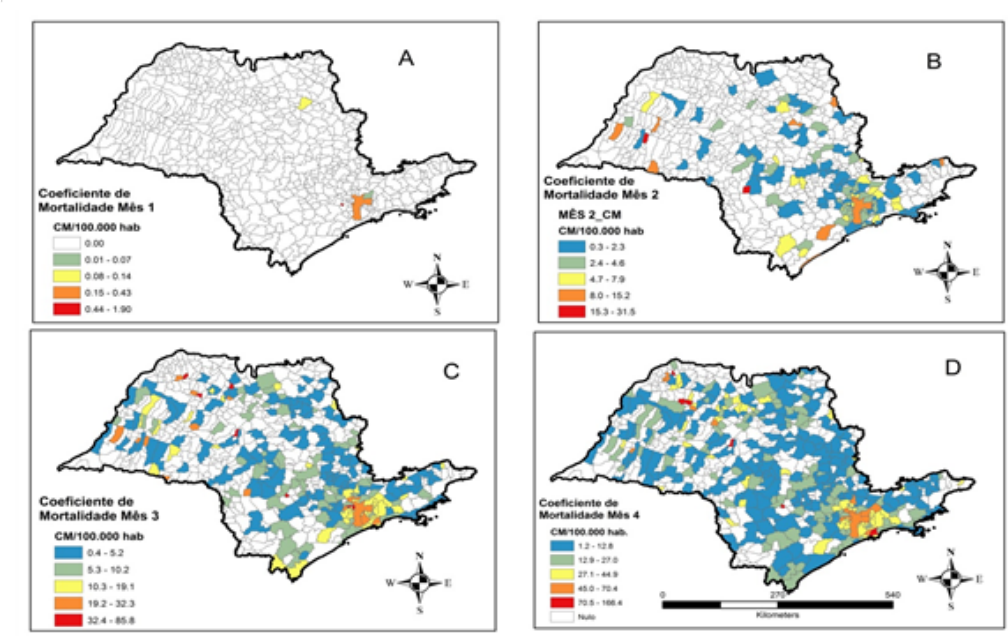

Fonte: Governo do Estado de São Paulo / Secretaria de Estado da Saúde / Coordenadoria de Controle de Doenças / Centro de Vigilância Epidemiológica "Prof. Alexandre Vranjac" - Novo Coronavírus (COVID-19) - Situação Epidemiológica / IBGE. registrado em 17 de março de 2020 no município de São Paulo e em 26 de junho o Estado de São Paulo confirmou 13.973 óbitos em 352 municípios (54,57\% dos 645 municípios). O município de São Paulo apresentou o maior número de óbitos $(6.880$ - 49,24\%) para o período. A variação dos óbitos entre os meses de março (57 óbitos) e abril (1.700 óbitos) foi de $2.982,46 \%$. Entre os meses de março e maio (5.868 óbitos) foi de 10.294,74\%, e entre os meses de março e junho (13.973 óbitos) foi de 24.514,04\% (Figura 3).

O coeficiente de mortalidade de COVID-19 acumulado para os quatro meses no Estado de São Paulo foi de 30,43 óbitos para cada 100.000 habitantes, sendo os municípios de maiores estimativas para esse indicador: Gastão Vidigal - 166,39; Dolcinopolis - 94,56; Uru - 85,84; Torres de Pedra - 82,92; Santos - 80,31; Nova Castilho - 78,93; Barueri - 70,39; Osasco - 67,87; Santa Salete - 64,72 e Cubatão - 63,50, todos para cada 100.000 habitantes, respectivamente (Figura 4).

A razão entre o número de óbitos e os casos confirmados (coeficiente de letalidade) acumulada para os quatro meses por COVID-19 no Estado de São Paulo foi de 5,41 para cada 100 casos. Os municípios de Álvaro de Carvalho, Coronel Macedo, Arapei, Turiuba, apresentaram a estimativa de $100 \%$ de letalidade por COVID-19. E os municípios de Alfredo Marcondes, Echapora, Iepe, Natividade da Serra, Poloni, Sabino, Sales, Santopolis do Aguapei tiveram a estimativa de $50 \%$ de letalidade para a doença (Figura 5).

\section{DISCUSSÃO}

A pandemia da COVID-19 pelo Novo Coronavírus (SARS-CoV-2) tem se apresentado como um dos maiores desafios sanitários em escala global deste início de século12. O Brasil apareceu como o segundo país em número de casos confirmados e de mortos (respectivamente 1.228.114 e 54.971 em 25 de junho de 2020)13, com destaque para o Estado de 
Figura 5 - Distribuição do coeficiente de letalidade de COVID-19 (A - 1 mês após o primeiro caso confirmado; B - 2 meses após o primeiro caso; C -3 meses após o primeiro caso e D - 4 meses após o primeiro caso), segundo município de residência. Estado de São Paulo, 26 de fevereiro a 26 de junho de 2020.
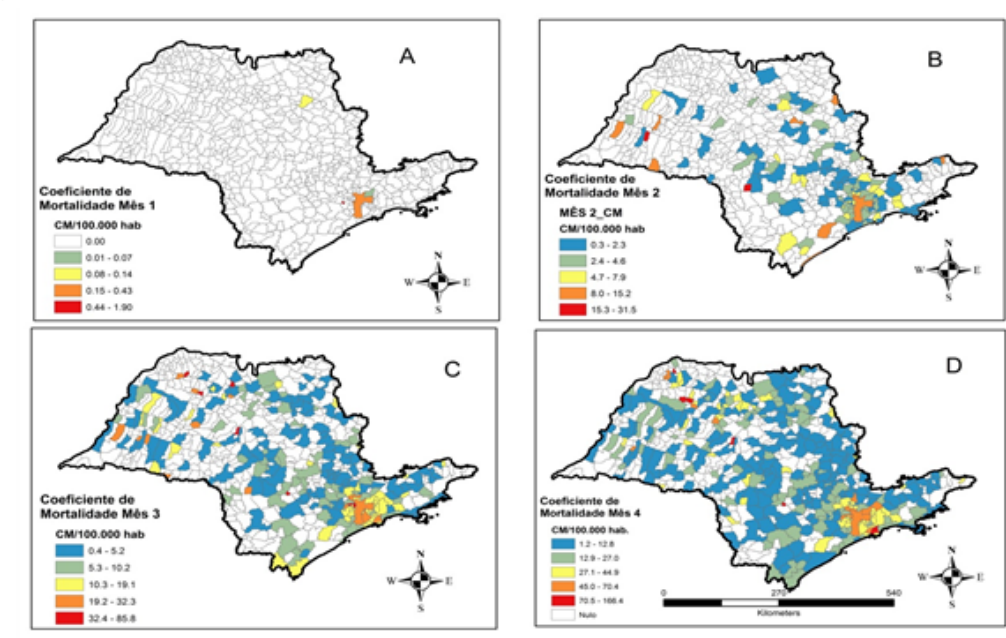

Fonte: Governo do Estado de São Paulo / Secretaria de Estado da Saúde / Coordenadoria de Controle de Doenças / Centro de Vigilância Epidemiológica "Prof. Alexandre Vranjac" - Novo Coronavírus (COVID-19) - Situação Epidemiológica / IBGE.

São Paulo.

O presente estudo, ao analisar a pandemia de COVID-19 no Estado de São Paulo, identificou que as estimativas dos coeficientes de incidência e de mortalidade para o período estudado foram, respectivamente, 562,96 e 30,43 para cada 100.000 habitantes até 26 de junho de 2020. Considerando todos os casos desde o início da pandemia até o dia 26 de junho, o coeficiente mundial de incidência foi 121,5 casos para 100.000 habitantes e o de mortalidade foi de 6,2 óbitos para cada 100.000 habitantes $^{13}$.

Devido à grande concentração populacional, as condições socioeconômicas mais díspares e regiões com grande população flutuante, as megacidades como São Paulo foram confrontadas com maiores surtos que tiveram importância na disseminação da infecção por COVID-19 ${ }^{14}$. Além disso, constata-se que as regiões próximas geograficamente tendem a apresentar coeficientes mais semelhantes do que as mais afastadas ${ }^{15}$. Assim, municípios próximos à capital apresentaram incidência e mortalidade altos, padrão que não foi observado para a letalidade.
A movimentação da população entre as cidades integrantes da Grande São Paulo e os municípios vizinhos é outro fator que favorece a disseminação da doença ${ }^{14}$.

Houve diferenças importantes entre os municípios que apresentaram as maiores magnitudes para cada indicador estudado. Doze municípios apresentaram estimativas dos coeficientes de incidência que superam a magnitude da capital no quarto mês da pandemia e, sendo estes, em ordem decrescente: Santos, Igaratá, Cubatão, Macatuba, Santa Salete, São João da Duas Pontes, Lencóis Paulista, Estrela d'Oeste, Jaci, Mendonça, Gurarujá e Tabapuã. As maiores estimativas dos coeficientes de mortalidade foram observados para Gastão Vidigal, Dolcinópolis, Santos, Nova Castilho, Barueri, Osasco, Santa Salete e Cubatão - cuja magnitude superou a observada para a capital do Estado no terceiro mês da pandemia - e Uru e Torres de Pedra - que mantiveram a magnitude superior à da capital para o indicador.

Ficaram em destaque, por terem altas magnitudes de incidência e mortalidade, os municípios de Santos, Santa Salete e Cubatão, e, devido ao fato de manterem posição de alta magnitude dos indicadores para os últimos dois meses de pandemia, os municípios de Santos, Uru e Torre de Pedras.

Nos países que apresentam amplas restrições tanto na capacidade de testagem nos momentos inicias da pandemia como na cobertura da assistência ao paciente grave, como os Estados Unidos e a Itália, o "isolamento vertical" (dos doentes e dos mais suscetíveis ao agravamento) foi inicialmente executado, porém, a evolução rápida do número de casos exigiu, ainda que tardiamente, a introdução da estratégia de supressão via "isolamento horizontal" (para a população, como um todo $)^{12}$.

No quarto mês da pandemia, as quatro cidades do Estado de São Paulo que apresentavam a estimativa de letalidade de $100 \%$ foram Coronel Macedo, Arapei, Turiuba e Álvaro de Carvalho, sendo que as três primeiras não tinham casos até 26 de maio. Para o mesmo período oito cidades tinham a estimativa de letalidade de $50 \%$, sendo estas: Alfredo Marcondes, Echaporã, Natividade da Serra, Sabino, Santópolis do Aguapei, Poloni, Sales e lepê.

A elevada estimativa do coeficiente de letalidade em 12 municípios pode ser justificada pelo fato de um número de casos da doença muito pequeno que evoluíram para óbito. Acredita-se também que o fato de a letalidade ser alta nesses municípios deve-se a subestimação do número de casos.

A detecção e a investigação dos casos são importantes, pois evidências sólidas, de boa qualidade, permitem o conhecimento do problema real, e assim o combate das iniquidades em saúde. Sem elas, a avaliação da equidade em saúde será impossível, o que reduzirá o impacto dos esforços de controle da doença. Além disso, garantir oportunidades de diagnóstico e tratamento para todos é visto como um fator importante para o enfrentamento e superação da doença ${ }^{16}$.

Os resultados deste estudo devem ser considerados à luz de algumas limitações. A maior limitação da análise de 
dados ecológicos conduzida no presente estudo tem relação com a heterogeneidade entre os municípios e populações em relação a exposição à doença ${ }^{15}$ e aos fatores que influenciam seu impacto como problema de saúde pública, como a estrutura etária da população, a extensão da investigação de casos, o acesso em tempo oportuno a serviços de saúde, adoção de medidas de isolamento social e de outras medidas de redução do risco de contágio não serem conhecidos. Tais fatores não foram incluídos nas análises.

Além disso, conduzir a comparação simples dos indicadores entre as regiões estudadas pode ser complexo, pois regiões com número de casos observados pequenos mostram grande variabilidade para os coeficientes estimados ${ }^{15}$. Assim, as estimativas dos coeficientes mais altos tendem a ser observados para municípios pequenos, como pôde ser observado, provavelmente, devido aos clusters de casos.

Finalmente, este trabalho apresentou limitações devido a utilização de dados secundários que, podem apresentar inconsistência em relação à quantidade, qualidade e processamento das informações. E como ponto forte, ao analisar as estimativas dos indicadores de saúde da COVID-19 no Estado de São Paulo ficou evidenciado áreas de maior risco para incidência, mortalidade e letalidade para o período estudado.

\section{CONCLUSÃO}

Após quatro meses da ocorrência do primeiro caso de COVID-19, a pandemia atingiu quase que a totalidade dos municípios do Estado, fato que pode ser justificado por diversos fatores, entre eles: o grande poder de disseminação da doença, a presença de uma população suscetível, por trata-se de um novo tipo de vírus, e da indisponibilidade de uma vacina.

O município de São Paulo figura nos quatro primeiros meses como a região mais atingida pela COVID-19, apresentando 121.163 casos confirmados e 6.880 óbitos. No entanto, segundo o "ranking" dos municípios no Estado, a respeito das estimativas dos indicadores de saúde de COVID-19, a capital fica na $13^{\text {a }}$ colocação quanto ao coeficiente de incidência $(988,92$ casos/100.000 habitantes) e no $12^{\circ}$ lugar em relação ao coeficiente de mortalidade (956,12 casos/100.000 habitantes).

Os resultados deste estudo sugerem auxílio aos gestores de saúde na tomada de decisões, haja vista que os mapas permitem visualizar os coeficientes de incidência, mortalidade e letalidade, permitindo assim a comparação entre as regiões, orientando na tomada de decisões para controle e prevenção da pandemia da COVID-19.

O conhecimento de áreas de potencial preocupação interfere no planejamento prévio e reuni recursos suficientes que são essenciais para a formulação de programas de saúde pública bem-sucedidos.

\section{Referências}

1. Lu H, Stratton CW, Tang YW. Outbreak of pneumonia of unknown etiology in Wuhan, China: The mystery and the miracle. J Med Virol. 2020;92(4):401-02. 2. Jung SM, Kinoshita R, Thompson RN, Linton NM, Yang Y, Akhmetzhanov AR, Nishiura H. Epidemiological Identification of A Novel Pathogen in Real Time: Analysis of the Atypical Pneumonia Outbreak in Wuhan, China, 2019-2020. J. Clin. Med. 2020;9(3):637.

3. Zhu N, Zhang D, Wang W, Li X, Yang B, Song J, Zhao X, Huang B, Shi W, Lu R, Niu P, Zhan F, Ma X, Wang D, Xu W, Wu G, Gao GF, Tan W. China Novel Coronavirus Investigating and Research Team. A Novel Coronavirus from Patients with Pneumonia in China, 2019. N Engl J Med. 2020;382(8):727-33.

4. World Health Organization. Statement on the second meeting of the International Health Regulations. Emergency Committee regarding the outbreak of novel coronavirus (2019-nCoV). Geneva: World Health Organization; 2020. Disponivel em: https://www.who.int/news-room/detail/30-01-2020-statement-on-the-second-meetingof-the-international-health-regulations-(2005)-emergency-committee-regarding-the-outbreak-of-novel-coronavirus-(2019-ncov), Acesso em 02/08/2020

5. Brasil. Ministério da Saúde. Portaria MS/GM n. 188, de 3 de fevereiro de 2020. Declara Emergência em Saúde Pública de importância Nacional (ESPIN) em decorrência da Infecção Humana pelo novo Coronavírus (2019-nCoV). Diário Oficial da União, Brasília (DF), 4 fev 2020; Seção 1:1. Disponível em: Disponivel em: http://www.in.gov.br/web/dou/-/portaria-n-188-de-3-de-fevereiro-de-2020-241408388. Acesso em 02/08/2020.

6. World Health Organization. WHO Director-General's opening remarks at the media briefing on COVID-19. 2020. Disponivel em https://www.who.int/dg/ speeches/detail/who-director-general-s-opening-remarks-at-the-media-briefing-on-covid-19---11-march-2020 . Acesso em 03/08/2020

7. World Health Organization. Coronavirus disease (COVID-19). Situation Report. 2020. Disponível em: https://www.who.int/docs/default-source/coronavi- ruse/situation-reports/20200928-weekly-epi-update.pdf?sfvrsn=9e354665_6. Acesso em 03/10/2020

8. Brasil. Ministério da Saúde. Secretaria de Vigilância em Saúde. Boletim Epidemiológico Especial 33. COE-COVID19. Brasília, 2020. Disponível em https:// portalarquivos2.saude.gov.br/images/pdf/2020/0ctober/01/Boletim-epidemiologico-COVID-33-final.pdf. Acesso em 03/10/2020.

9. The Lancet. COVID-19 in Brazil: "So what". The Lancet, 395(10235), 14611462. Disponivel em https://doi.org/10.1016/S0140-6736(20)31095-3. Acesso em 01/08/2020.

10. Macedo YM, Ornellas JL, Bomfim HF. COVID - 19 no Brasil: o que se espera para população subalternizada? Revista Encantar - Educação, Cultura e Sociedade - Bom Jesus da Lapa; 2020(2)1-10.

11. Rodriguez-Morales AJ, Gallego V, Escalera-Antezana JP, Méndez CA, Zambrano LI, Franco-Paredes C, Suárez JA, Rodriguez-Enciso HD, Balbin-Ramon GJ, Savio-Larriera E, Risquez A, Cimerman S. COVID-19 in Latin America: The implications of the first confirmed case in Brazil. Travel Med Infect Dis.; 2020(35):101613.

12. Werneck GL, Carvalho MS. A pandemia de COVID-19 no Brasil: crônica de uma crise sanitária anunciada. Cad Saúde Pública. 2020; 36(5):e00068820. 13. World-o-Meter. Disponível em: https://www.worldometers.info/coronavirusl. Acesso em: 29/07/2020.

14. Ren H, Zhao L, Zhang A, Song L, Liao Y, Lu W, Cui C. Early forecasting of the potential risk zones of COVID-19 in China's megacities. Science of The Total Environment. 2020;729.

15. Morgenstern H. Ecologic Studies in Epidemiology: Concepts, Principles and Methods. Annu Rev Public Health. 1995;16:61-81.

16. Figueiredo AM, Codina AD, de Figueiredo DCMM, Gil-García E, Kalache A. Letalidad del COVID-19: ausencia de patron epidemiológico. Gaceta Sanitaria (2020). 